\title{
Sublinear Message Bounds for Randomized Agreement
}

\author{
John Augustine* \\ Department of Computer Science and \\ Engineering, Indian Institute of \\ Technology at Madras \\ Chennai, Tamil Nadu, India \\ augustine@iitm.ac.in
}

\author{
Anisur Rahaman Molla ${ }^{\dagger}$ \\ School of Computer Sciences, \\ National Institute of Science \\ Education and Research Bhubaneswar \\ Khurda, Odisha, India \\ molla@niser.ac.in
}

\author{
Gopal Pandurangan ${ }^{\ddagger}$ \\ Department of Computer Science, \\ University of Houston \\ Houston, Texas, USA \\ gopalpandurangan@gmail.com
}

\begin{abstract}
This paper focuses on understanding the message complexity of randomized agreement in synchronous distributed networks. We focus on the so-called implicit agreement problem where each node starts with an input value (0 or 1$)$ and at the end one or more nodes should decide on a common input value which should be equal to some node's input value (there can be undecided nodes). Implicit agreement is a generalization of the fundamental agreement and leader election problems.

We present sublinear (in $n$, where $n$ is the number of nodes) algorithms and lower bounds on the message complexity of implicit agreement in fully-connected (i.e., complete) networks. Specifically our main results are:
\end{abstract}

(1) We show that for any implicit agreement algorithm that succeeds with probability at least $1-\epsilon$, for some suitably small constant $\epsilon>0$, needs at least $\Omega\left(n^{0.5}\right)$ messages with constant probability. This bound holds regardless of the number of rounds used and applies to both LOCAL and CONGEST models. This lower bound is essentially tight for complete networks, as there exists a randomized agreement algorithm that uses only $\tilde{O}\left(n^{0.5}\right)$ messages $^{1}$ with high probability ${ }^{2}$ and runs in $O(1)$ rounds and succeeds with high probability. Both the upper and lower bounds assume that nodes have access to (only) private coins.

(2) In contrast to the above bounds, if nodes have access to an unbiased global (shared) coin, we present a randomized algorithm which, with high probability, achieves implicit agreement, and uses $\tilde{O}\left(n^{0.4}\right)$ messages in expectation and runs

${ }^{*}$ Research supported in part by an Extra-Mural Research Grant (file number EMR/2016/003016) funded by the Science and Engineering Research Board, Department of Science and Technology, Government of India.

${ }^{\dagger}$ Supported by DST Inspire Faculty Research grant (grant number DST/INSPIRE/04/2015/002801).

¥ Supported, in part, by NSF grants CCF-1527867, CCF-1540512, IIS-1633720, CCF-BSF1717075, and BSF award 2016419.

${ }^{1}$ The notations $\tilde{O}$ and $\tilde{\Omega}$ hide polylog $(n)$ factors.

${ }^{2}$ Throughout,"with high probability" or "whp" in short means with probability at least $1-1 / n^{c}$ for some constant $c \geq 1$.

Permission to make digital or hard copies of all or part of this work for personal or classroom use is granted without fee provided that copies are not made or distributed for profit or commercial advantage and that copies bear this notice and the full citation on the first page. Copyrights for components of this work owned by others than the author(s) must be honored. Abstracting with credit is permitted. To copy otherwise, or republish, to post on servers or to redistribute to lists, requires prior specific permission and/or a fee. Request permissions from permissions@acm.org.

PODC'18, July 23-27, 2018, Egham, United Kingdom

(C) 2018 Copyright held by the owner/author(s). Publication rights licensed to ACM ACM ISBN 978-1-4503-5795-1/18/07 . \$ $\$ 15.00$

https://doi.org/10.1145/3212734.3212751 in $O(1)$ rounds (deterministically). This algorithm works in the CONGEST model as well. Our result shows the power of a global coin in significantly improving (by a polynomial factor) the message complexity of agreement. As another contrast, we show that the same benefit does not apply to leader election, i.e., even with access to a global coin, $\Omega\left(n^{0.5}\right)$ messages (in expectation) are needed for any leader election algorithm that succeeds with probability at least $1-\epsilon$, for a small constant $\epsilon>0$.

(3) We extend our results to a natural generalization of agreement called as subset agreement where a given (non-empty) subset of nodes should agree on a common value. We show that subset agreement on a subset of size $k$ nodes can be accomplished by a randomized algorithm that succeeds with high probability, and uses (in expecation) $\tilde{O}\left(\min \left\{k n^{0.5}, n\right\}\right)$ (using only private coins) and $\tilde{O}\left(\min \left\{k n^{0.4}, n\right\}\right)$ messages (using global coin) respectively.

We view our results as a step towards understanding the complexity of randomized agreement in distributed networks, in particular, message complexity with or without shared randomness.

\section{CCS CONCEPTS}

- Theory of computation $\rightarrow$ Design and analysis of algorithms; Models of computation; ・ Networks $\rightarrow$ Network algorithms;

\section{KEYWORDS}

Distributed Algorithm, Randomized Algorithm, Agreement, Leader Election, Global Coin, Shared Randomness

\section{ACM Reference Format:}

John Augustine, Anisur Rahaman Molla, and Gopal Pandurangan. 2018. Sublinear Message Bounds for Randomized Agreement. In PODC '18: ACM Symposium on Principles of Distributed Computing, Fuly 23-27, 2018, Egham, United Kingdom. ACM, New York, NY, USA, 10 pages. https://doi.org/10. $1145 / 3212734.3212751$

\section{INTRODUCTION}

Agreement is a fundamental problem in distributed computing and, along with its variants, it has been extensively studied for the last four decades. In this paper, we focus on a basic question in solving agreement in (synchronous) distributed networks: What is the minimum number of messages required to solve agreement? In the classical (binary) agreement problem, we have $n$ nodes each starting with an input value ( 0 or 1$)$ and the goal of agreement is to guarantee the following two conditions: (1) All nodes should agree (decide) on the same input value (consensus condition) and (2) the 
common value should be the input value of some node (validity condition). If there are no failures, the above problem is easy to solve. For example, in a fully-connected (i.e., complete) network with all good nodes, the following simple algorithm takes only 1 round: each node broadcasts its value to all other nodes and then all nodes take the majority value to be consensus value (if it is a tie, then they can all choose, say, 1). However, this easy solution, though optimal in the number of rounds, requires $\Theta\left(n^{2}\right)$ messages. ${ }^{3}$

The problem becomes much more difficult to solve when there are faults or Byzantine failures; these have been extensively investigated and continue to be investigated with a long history (e.g., see $[5,18]$ or the more recent results discussed below). In the vast majority of the works in the literature (under various settings and assumptions), the focus is mainly on the time complexity, i.e., the number of rounds needed for agreement. The message complexity has also been investigated, but somewhat less number of results have focused on this aspect. To be concrete, let's focus on the Byzantine agreement problem (on fully-connected networks) which was first defined in early 80 's by a landmark paper by Pease, Shostak, and Lamport [23] and has since been studied extensively. In the Byzantine setting, a subset of the nodes can be Byzantine, i.e., can behave maliciously (including crashing) and can deviate arbitrarily from the behaviour of the protocol executed by the good nodes and try to foil the good nodes reaching agreement. In the Byzantine setting, condition (1) should hold for all honest nodes, i.e., all honest nodes should agree on a common value (condition 2 also holds). Byzantine agreement can be considered as a "flagship" problem in distributed computing, as it gives a building block of creating reliable distributed systems that can operate under failures and malicious nodes. Its importance can be seen from the widespread and continued application in many domains, e.g., sensor networks [27], grid computing [4], peer-to-peer networks [26] and cloud computing [28]. However, despite intensive research, there has still not been a practical solution to the Byzantine agreement problem for large networks. A main reason for this is the large message complexity of currently known protocols; this has been suggested by many systems papers $[2,3,9,19,29]$. Till recently, the best known protocols had quadratic message complexity, i.e., $\Theta\left(n^{2}\right)$, where $n$ is the number of participants (nodes) in the network.

In a breakthrough result, King and Saia [12] presented the first Byzantine agreement algorithm in synchronous complete networks that breaks the quadratic message barrier. They presented an algorithm where each processor sends only $\tilde{O}(\sqrt{n})$ messages, and thus the total message complexity is bounded by $\tilde{O}\left(n^{1.5}\right)$. Their algorithm is randomized Monte Carlo (succeeds with high probability) and works even under an adaptive adversary which can control up to $1 / 3$ of the processors (but assumes private channels). This is essentially the best message complexity known for Byzantine agreement. However, no non-trivial message lower bound is known for Byzantine agreement. In particular, Saia and King raise the open question whether each processor needs to send at least $\tilde{\Omega}(\sqrt{n})$ to solve Byzantine agreement. Also, it is not known if one needs $\tilde{\Omega}\left(n^{1.5}\right)$ (total) message complexity. In fact, a tight message complexity of solving just agreement (even with no Byzantine nodes)

\footnotetext{
${ }^{3}$ Our results (cf. Section 1.1) implies that this problem can be solved optimally with regards to both time and message complexity by a randomized algorithm that uses $O(n)$ messages with high probability and runs in $O(1)$ rounds.
}

is still not fully understood in the randomized setting (cf. Section 1.3); this is especially true for the variants of the agreement problem addressed here (discussed below). Understanding the message complexity of randomized agreement in the fault-free setting will help shed light in the more challenging Byzantine setting; in particular, the lower bounds in the fault-free setting also apply for the Byzantine setting. This is one main motivation for this work.

Another main motivation for this work is understanding the significance of a key resource in randomized agreement, namely, the use of private versus global (shared) coins. Protocols that use private coins only, assume that all nodes have access to private (unbiased) coin tosses that are not visible to other nodes. On the other hand, protocols that use global coins assume that all nodes have access to a shared coin. For example, one of the first randomized protocols for Byzantine agreement by Rabin [25] (see the protocol's description in [21]) uses an unbiased global coin to achieve Byzantine agreement in (expected) constant number of rounds in a complete network; however, this protocol takes $\Theta\left(n^{2}\right)$ messages. Many other randomized protocols have been designed since then that uses private coins. One of the earliest protocols by Ben-Or [6] also uses private coins, but this also takes $\Theta\left(n^{2}\right)$ messages (and also exponential time to finish if there is a linear number of Byzantine nodes). There has been a lot of work on "coin flipping" protocols (see e.g., [7] and the references therein) where the goal is to generate a common coin despite the presence of Byzantine nodes. Common coin (see e.g., $[8,10]$ for a definition) is a somewhat weaker notion than a global coin where it is sufficient for the value to be the same across all (honest) nodes with only constant probability and both 0 and 1 values should occur with constant probability. Common coins are a fundamental primitive that enables implementation of randomized byzantine agreement (among other protocols), e.g., it is sufficient for implementing Rabin's protocol [8]. However, almost all the work till now has focused on reducing the time complexity of coin flipping and not the message complexity. All these protocols have $\Theta\left(n^{2}\right)$ message complexity (except for the work of King and Saia [12] discussed earlier which has $\tilde{O}\left(n^{1.5}\right)$ message complexity). Hence a fundamental question is understanding the message complexity of coin flipping as well as the communication (message) cost of using global coin verses private coins.

In this paper, motivated by understanding the message complexity of randomized agreement, we introduce and study the notions of implicit agreement and subset agreement which generalize the agreement problem. Intuitively, an "implicit" agreement doesn't require all the participants to agree on a value, rather it is enough if a non-empty subset of them agree. Formally we define implicit agreement on an $n$ node-network as follows.

Definition 1.1 (Implicit Agreement). Suppose initially each node has an input value in $\{0,1\}$. An implicit agreement holds when the final state of all the nodes is either $\{0, \perp\}$ or $\{1, \perp\}$ and at least one node has state other than $\perp$ (which should be the input value of some node), where $\perp$ denotes the 'undecided' state. In other words, all the decided nodes must agree on the same value which is an initial input value of some node and there must be at least one decided node in the network. 
We study the implicit agreement problem in a fully-connected (complete) $n$-node distributed network. Implicit agreement is a generalization of the agreement problem. The flexibility of the implicit agreement lies in the fact that all the nodes need not agree or do not need to know the agreed value. Therefore, in principle, the agreement can be fulfilled with much less communication. In particular, sublinear message bounds are possible, unlike the case of the usual agreement when all nodes need to agree (this requires at least $n$ messages). Implicit agreement is also interesting in understanding lower bounds for agreement in the faulty setting (either crash failures or Byzantine nodes) where we don't care about the values output by the "bad" nodes. Thus, clearly, lower bounds for implicit agreement apply for full agreement in the faulty setting as well. Implicit agreement also generalizes the fundamental leader election problem, or, in other words, leader election can be considered as a special case of implicit agreement. We note that if there is exactly one decided node, then the problem is equivalent to implicit leader election problem which has been studied extensively for over three decades, see e.g., $[5,16-18]$ and the references therein (see also Section 1.3). The decider node will be the leader. However, in comparison to the leader election problem, the implicit agreement has the flexibility of multiple nodes deciding (and hence presumably easier), however, the challenging part is that all the deciding nodes must decide on the same value (which should be the input value of some node as well). In this paper, we also fully characterize the message complexity of (implicit) leader election vis-a-vis implicit agreement.

We also consider a natural generalization of implicit agreement called subset agreement which can also be independent interest and useful in applications. For example, consider a large network such as the Internet, and an (a priori) unknown subset of nodes want to agree on a common value; the subset size can be much smaller than the network size.

Definition 1.2 (Subset Agreement). Initially each node has a value in $\{0,1\}$, the subset agreement is an agreement by a (specified) non-empty subset $S \subseteq V$ of nodes. We assume that each node knows whether it belongs to $S$ or not, but doesn't know the identities of other nodes in the subset. The agreement on $S$ holds when the final state of all the nodes in $S$ is "decided" and the deciding value of all of them is either 0 or 1 (which should be the input value of some node in the network).

Note that subset agreement is the same as traditional agreement if the subset includes all the nodes. We study sublinear message bounds for subset agreement.

\subsection{Our Contributions}

We present sublinear (in $n$, where $n$ is the number of nodes) algorithms and lower bounds on the message complexity of implicit agreement and subset agreement in fully-connected (i.e., complete) networks. Specifically our main results are:

(1) We show that for any implicit agreement algorithm that succeeds with probability at least $1-\epsilon$, for some suitably small constant $\epsilon>0$, needs at least $\Omega\left(n^{0.5}\right)$ messages with constant probability. (Note that this implies that $\Omega\left(n^{0.5}\right)$ messages are needed in expectation as well.) This lower bound is essentially tight for complete networks, as there exists a randomized algorithm for implicit agreement that uses only $\tilde{O}\left(n^{0.5}\right)$ messages with high probability ${ }^{4}$ and runs in $O(1)$ rounds and succeeds with high probability. ${ }^{5}$ Both the upper and lower bounds assume that nodes have access to (only) private coins.

(2) In contrast to the above bounds, if nodes have access to an unbiased global (shared) coin, we present a randomized algorithm for implicit agreement which succeeds with high probability, and that uses $\tilde{O}\left(n^{0.4}\right)$ messages (in expectation) ${ }^{6}$ and runs in $O(1)$ rounds (deterministically). Our result shows the power of a global coin in significantly improving (by a polynomial factor) the message complexity of implicit agreement. As another contrast, we show that the same benefit does not apply to leader election, i.e., even with access to a global coin, $\Omega\left(n^{0.5}\right)$ messages are needed for any leader election algorithm that succeeds with probability at least $\epsilon$, for a fixed constant $0<\epsilon<1$. Our results show that agreement is a strictly easier problem (with respect to message complexity) than leader election under access to global coin.

(3) We show that subset agreement on a subset of size $k$ nodes can be accomplished by randomized algorithm, which succeeds with high probability, and uses (in expectation) $\tilde{O}\left(\min \left\{k n^{0.5}, n\right\}\right)$ messages (with access to private coins only) and $\tilde{O}\left(\min \left\{k n^{0.4}, n\right\}\right)$ messages (with access to global coin). We note that these bounds are sublinear in $n$ when $k=o(\sqrt{n})$ and $k=o\left(n^{0.6}\right)$ respectively.

For our algorithms, we assume that the communication is synchronous and follows the standard CONGEST model [24], where a node can send in each round at most one message of size $O(\log n)$ bits on a single edge. We do not however assume that the nodes have unique IDs, hence the algorithms in this paper apply also for anonymous networks. We assume that all nodes wake up simultaneously at the beginning of the execution. (Additional details on our distributed computation model are given in Section 1.2.) Our lower bounds hold regardless of the number of rounds even in the LOCAL model [24], where there is no restriction on the number of bits that can be sent on each edge in each round.

We view our results as a step towards understanding the complexity of randomized agreement in distributed networks, in particular, message complexity with or without shared randomness. Our work raises many interesting open problems which we discuss in Section 6.

\subsection{Distributed Computing Model}

The distributed computing model we consider is similar to the models of $[1,11,13-15]$, with the main addition of giving processors access to a private unbiased coin. For our algorithm of Section 3, we also allow processors access to a global (shared) unbiased coin. Also, we do not assume unique identities; however, we show that our lower bounds hold regardless of whether we have unique identities or not. We consider a network of $n$ nodes, represented as

\footnotetext{
${ }^{4}$ Throughout, "with high probability" or "whp" in short means with probability at least $1-1 / n^{c}$ for some constant $c \geq 1$.

${ }^{5}$ It can be shown that, if no randomness is allowed, i.e., for deterministic algorithms, $\Omega(n)$ is a (tight) lower bound on the message complexity of implicit agreement.

${ }^{6}$ The message complexity bound also holds with probability at least $1-1 / n^{1 / 5}$
} 
an undirected complete (fully-connected) graph $G=(V, E)$. Each node runs an instance of a distributed algorithm. The computation advances in synchronous rounds where, in every round, nodes can send messages, receive messages that were sent in the same round by neighbors in $G$, and perform some local computation. Messages are the only means of communication; in particular, nodes cannot access the coin flips of other nodes, and do not share any memory. Throughout this paper, we assume that all nodes are awake initially and simultaneously start executing the algorithm.

We note that initially nodes have knowledge only of themselves, in other words we assume the clean network model - also called the KT0 model [24] which is standard and most commonly used. On the other hand, if one assumes the KT1 model, where nodes have an initial knowledge of the IDs of their neighbors, then leader election (and hence implicit agreement) is trivial, since the minimum ID node can become the leader. (However, subset agreement is still not trivial if nodes in the subset don't know each other, as assumed here.)

\subsection{Other Related Work}

We focus only on those works that are closely related to the work here, in particular, agreement and leader election in the fault-free setting. The work of Kutten et al. [17, 22] considers the (implicit) leader election in complete networks and showed that, contrary to deterministic setting (which has been studied extensively see e.g., [17] and the references therein), randomization allows one to obtain sublinear message bounds. In particular, they demonstrated that, for randomized algorithms, the seemingly obvious lower bound of $\Omega(n)$ messages for a complete graph does not hold. Specifically, it presented an algorithm that executes in $O(1)$ time and uses only $O\left(\sqrt{n} \log ^{3 / 2} n\right)$ messages to elect a leader in a complete graph. They also showed an almost matching lower bound for randomized leader election, by showing that $\Omega(\sqrt{n})$ messages are needed for any leader election algorithm that succeeds with probability at least $1 / e+\epsilon$, for any small constant $\epsilon>0$. The subsequent work of Kutten et al. [16] studied (implicit) leader election in general networks and showed that $\Theta(m)$ ( $m$ is the number of edges) and $\Theta(D)(D$ is the network diameter) are tight bounds on the message and time complexity of randomized leader election in general networks.

The present work is inspired by the works of $[16,17]$ and studies the randomized complexity of (implicit) agreement. It is clear that an algorithm for leader election also solves implicit agreement, but since agreement is weaker, a natural question is whether the $\Omega(\sqrt{n})$ message lower bound for leader election also applies for agreement. We show that it indeed does, although the proof for the agreement is different and more involved (the main reason being that the agreement problem has inputs, unlike, leader election). This motivates us to consider agreement with global coins where we show that the $\Omega(\sqrt{n})$ lower bound can be beaten by a polynomial factor: we can obtain a $\tilde{O}\left(n^{0.4}\right)$ messages algorithm. This is in contrast to leader election where we show that having access shared coins does not help: the $\Omega(\sqrt{n})$ lower bound still holds. This proof closely builds on the lower bound proof of [17].

To the best of our knowledge, the subset agreement problem has not been studied before.

\section{LOWER BOUND FOR IMPLICIT AGREEMENT}

In this section, we show that any algorithm for implicit agreement - even randomized - will, with constant probability, require $\Omega(\sqrt{n})$ messages to be sent in order to achieve implicit agreement with probability at least $1-\epsilon$ for a sufficiently small $\epsilon>0$. Kutten et al. [17] show that leader election requires $\Omega(\sqrt{n})$ messages, but our proof is different because, under leader election, all nodes essentially start in identical configurations. In our implicit agreement problem, however, the nodes start with initial values and our lower bound argument takes that into account and shows that no algorithm can exploit those initial values in order to reach agreement with fewer messages.

Consider a randomized algorithm $\mathcal{A}$ to achieve implicit agreement with probability $1-\epsilon$. For the sake of contradiction, let us assume that $\mathcal{A}$ sends at most $o(\sqrt{n})$ messages with probability at least $1-o(1)$. $\mathcal{A}$ can be randomized in the sense that each node individually has access to a source of uniform and independent random bits. We will show that $\mathcal{A}$ must have an error probability that is at least a constant, so for the sake of contradiction, assume that the probability with which $\mathcal{A}$ does not reach implicit agreement is at most $o(1)$. For now, we will assume that the nodes are anonymous, so $\mathcal{A}$ does not have access to unique node identifiers or any other identifying feature pertaining to individual nodes.

Recall also that the network model is the KT0 variant, so a node $u$, at least at the start of the protocol, will not know which incident edge leads to some neighbour $v$. In fact, for the purpose of showing this lower bound, we assume that for every node, the neighbor sequence (as we enumerate from port 1 to port $n-1$ ) is a uniformly random permutation independent of all other nodes' permutations.

Given any $p \in[0,1]$, let $C_{p}$ denote the (random) starting configuration wherein each node is independently assigned an initial value of 1 with probability $p$, or 0 with probability $1-p$. Let $G_{p}$ be the (random) directed graph on the $n$ nodes with an edge from $u$ to $v$ iff $u$ sent a message to $v$ and the message was sent before $v$ sent any message to $u$ as $\mathcal{A}$ executed from the starting configuration $C_{p}$. We now show that with probability at least $1-\epsilon, G_{p}$ is a forest comprising trees that are directed away from their respective root.

LEMMA 2.1. With probability at least $1-\epsilon^{\prime}$ for some arbitrarily small but constant $\epsilon^{\prime}>0$, the graph $G_{p}$ for all $p \in[0,1]$ is a forest in which each tree contains exactly one node (called its root) with zero in-edges and, furthermore, all tree edges are oriented away from the root.

Proof. Recall that the number of messages passed by $\mathcal{A}$ is at most $o(\sqrt{n})$ with probability at least $1-o(1)$. Therefore, with probability at least $1-o(1)$, we can apply the condition that the number of nodes that participate in any form of communication (either sending or receiving) is at most $o(\sqrt{n})$. Recall also that each message is sent to a random node. Therefore, the probability that none of those $o(\sqrt{n})$ messages is targeted towards either a node that has sent messages or a node that has already received some message is at least $(1-o(\sqrt{n}) / n)^{o(\sqrt{n})} \geq 1-o(1)$. Removing the conditioning, we get the probability that all components in $G_{p}$ are rooted and oriented trees is again at least $1-\epsilon^{\prime}$ for some fixed $\epsilon^{\prime}>0$. 
Thus, for the rest of the argument, we apply the condition that for all $p \in[0,1], G_{p}$ is a forest as described in Lemma 2.1. We say that a tree is a deciding tree if there is at least one deciding node within that tree.

Lemma 2.2. For every value of $p \in[0,1]$, the probability that there are at least two deciding trees is at least a constant.

Proof. Since $\mathcal{A}$ reaches agreement with probability at least $1-\epsilon$, the probability that no tree decides is at most $\epsilon$.

For all values of $p$, the probability that there is at most one deciding tree is at most a suitable constant $c$ bounded away from 1. Otherwise there exists a $p$ value for which, with probability at least $c$, exactly one tree decides. Then, quite easily, the root of that deciding tree can be made a leader. This yields a leader election protocol that succeeds with probability at least $c$, which will contradict Theorem 3 from [17] when $c$ is a sufficiently large constant strictly less than $1-\epsilon$.

Adding up all these possibilities, we are still left with a probability of at least a constant $q<1-\epsilon-c$ with which there must be two or mode deciding trees.

Now that we know that the there are at least two deciding trees with constant probability, we show that these trees reach opposing decisions with probability also bounded from below by a constant, thereby leading to a contradiction that $\mathcal{A}$ reaches agreement with high probability.

LEMMA 2.3. There exists a value $p^{*} \in[0,1]$ such that the probability that there are at least two deciding trees in $G_{p^{*}}$ with opposing decisions is at least a constant.

Proof. We can define the probabilistic valency of $p$ with respect to $\mathcal{A}$, denoted $V_{p}$, as the probability that $\mathcal{A}$ will terminate with a decision value 1 under the initial configuration $C_{p}$. Thus, the probabilistic valencies of $p=0$ and $p=1$ are 0 and 1 , respectively. It is easy to see that $V_{p}$ is a continuous function of $p \in[0,1]$ as infinitesimally small changes to $p$ can only result in infinitesimally small changes to $V_{p}$. This is true because infinitesimally small changes to $p$ will only result in infinitesimally small changes to $C_{p}$, and since the algorithm's behaviour is based on the initial configuration (which does not change much), $V_{p}$ will also only change infinitesimally. This continuity in $V_{p}$ implies that for every $x \in[0,1]$, there is a $p$ such that $V_{p}=x$.

We prove this lemma under the condition that there are at least two deciding trees and that these two trees do not interact with each other. We have seen that enforcing this conditioning requires a probability of $q$ bounded away from 0 . An important implication here is that, despite this conditioning, the probabilities of the two decision values must both be bounded from below by a constant. For the sake of contradiction, let us suppose (without loss of generality) that the algorithm decides 1 with probability at most $o(1)$ under this conditioning. Let us fix $p$ such that $V_{p}=1-q / 2$. Then, the probability that it decides 1 without the conditioning will be at most $q(o(1))+(1-q)<1-q / 2$, a contradiction.

Now consider two deciding trees $T_{1}$ and $T_{2}$. For simplicity, let us assume that the decision outcome of $T_{1}$ is (say) 0 ; this occurs with some constant probability. We now reason that the probability that the decision outcome of $T_{2}$ is 1 will be at least a constant. This is true because the random input values for $T_{2}$ were all chosen independent of the input values for $T_{1}$. Thus, the two trees can decide contradictory values with probability at least a constant.

Thus, we have shown that when the nodes are anonymous and the number of messages sent is at most $o(\sqrt{n})$, implicit agreement is not reached with probability at least a constant. To generalize to the case where nodes do have IDs, we simply assume that the adversary provides ID's chosen uniformly at random from, say, $\left[1, n^{4}\right]$. Let $\mathcal{A}^{*}$ be an algorithm that exploits these unique IDs.

Notice however, that the execution of $\mathcal{A}^{*}$ that exploits the adversarially generated random IDs is essentially akin to the execution of $\mathcal{A}^{*}$ in the anonymous setting in which each node first generates a random number between $\left[1, n^{4}\right]$ and then uses that random number as their IDs. The only difference is that multiple nodes may generate the same random number, thereby inheriting the same ID, but this is an event whose probability is at most $1 / n$, so when we condition on these random numbers being distinct, the two executions are probabilistically identical, so any lower bound on the execution without adversarially generated IDs will extend to the case where nodes have unique IDs provided by the adversary. Thus,

Theorem 2.4. Suppose $\mathcal{A}$ is an algorithm that solves implicit agreement with probability at least $1-\epsilon$ for some sufficiently small constant $\epsilon>0$ in a network of n nodes with unique IDs. Then, with probability at least a constant, the message complexity of $\mathcal{A}$ is at least $\Omega(\sqrt{n})$.

Optimal Message Complexity Algorithm: Kutten et al. [17, 22] present an algorithm that solves the leader election with high probability and uses $\tilde{O}(\sqrt{n})$ messages. Their algorithm immediately solves the implicit agreement problem as the leader decides on its own value. The following theorem on implicit agreement follows from their leader election result (Theorem 1, [17]).

THEOREM 2.5. With high probability, implicit agreement can be solved in $O(1)$ rounds and using at most $O\left(\sqrt{n} \log ^{3 / 2} n\right)$ messages with high probability on a complete n-node network.

\section{IMPLICIT AGREEMENT USING A GLOBAL COIN}

Let us now consider the case where the nodes have access to an unbiased global coin through which they can generate common random bits. The shared random bits are oblivious to the adversary, but it knows the algorithm. With the knowledge of the algorithm, the adversary determines the initial distribution of the $0-1$ values over the $n$ nodes. We study the implicit agreement problem under this additional assumption of shared random bits. We design a randomized algorithm which solves the implicit agreement problem with high probability in $O(1)$ communication rounds and incurs expected $O\left(n^{0.4} \log ^{8 / 5} n\right)$ messages. Note that our algorithm is correct, i.e., achieves agreement, with probability at least $1-1 / n$; however the message bound holds in expectation and also with probability at least $1-1 / n^{c}$, for some constant $c>0$, and the time bound of $O(1)$ is deterministic. 
High-level idea of the algorithm. We first select a smaller set of nodes which will be responsible for the agreement. This is done by choosing a random set of candidate nodes of size $O(\log n)$. For this, every node selects itself with probability $O(\log n / n)$ to become a candidate node. The candidate nodes are responsible for the agreement. Next, each candidate node $v$ samples another $O(\log n)$ random nodes from the network and gets their (input) values ( 0 or 1$)$. Let $\mathrm{x}_{v}$ be the number of $1 \mathrm{~s}$ among $O(\log n)$ sampled values. Each candidate node $v$ computes $p(v)$ as the fraction of $1 \mathrm{~s}$ it receives, i.e., $p(v)=\mathrm{x}_{v} / \log n$. We show that with high probability $p(v) \in[\ell, \ell+5 / \sqrt{\log n}]$ for any candidate node $v$, where $\ell \in[0,(1-5 / \sqrt{\log n})]$. In other words, all the $p(v)$ values belong to a strip (an interval in $[0,1])$ ) of length $5 / \sqrt{\log n}$ (follows from the Lemma 3.1). Let us denote the strip by $\delta$. We abuse the notation by referring to the length of the strip by $\delta$ also. The candidate nodes then use the global coin and draw a random real number, say $r$, in the interval $[0,1]$. Notice that the value $r$ is same for the candidates nodes as they use same random bits (from the global coin). One simple way to generate a random (real) number ${ }^{7}$ in the interval $[0,1]$ from the random bit string is as follows. Suppose the random bit string is $S$. Then the random number is the converted decimal number of $0 . S$ (in binary). ${ }^{8}$ Then the candidate nodes decide on ' 0 ' if $p(v)$ is on the left of $r$; otherwise, they decide on ' 1 '. The only case where the agreement could be wrong is when the random number $r$ falls within the strip $\delta$ and the probability of that case is $\delta$ (note that this depends on the input values which is not under the control of the algorithm). The probability that the random number $r$ doesn't fall in the strip $\delta$ is $(1-\delta)$. Therefore, with probability $(1-5 / \sqrt{\log n})$, the real number $r$ is either on the left or on the right side of all the $p(v)$ values in the line $[0,1]$. That is with probability $(1-5 / \sqrt{\log n})$, all the candidate nodes decide on the same value and hence, the implicit agreement is satisfied. The message complexity of the algorithm is $O\left(\log ^{2} n\right)$ as the size of the candidate nodes is $O(\log n)$ and each candidate node contacts $O(\log n)$ random nodes. Thus, although this uses only polylogarithmic messages in total, the success probability is not high as required, i.e., it is not with probability $>(1-1 / n)$.

Note that in the above algorithm if we increase the number of sampled nodes by each candidate node then the strip length $\delta$ will be smaller and hence the probability that the random number $r$ will fall in the strip is smaller. That is, the probability of success increases if the number of samples is large. However, this doesn't help much as the number of samples counts towards the message complexity.

We now discuss how to achieve high probability of success. Recall that when the random number $r$ falls inside the strip $\delta$, then some candidate nodes may decide on 0 and some on 1 (based on left or right of $r$ ) and hence the agreement could be invalid. To get rid of this, we put some candidate nodes in undecided state, say to those nodes $v$ for which $|p(v)-r| \leq 4 \delta$. But then the issue is how the undecided (candidate) nodes know that there is at least

\footnotetext{
${ }^{7}$ For simplicity in the argument, we will assume a real number; however, we only need to sample $O(\log n)$ random bits from the shared coin to get a high-enough precision that is sufficient for the analysis. The error introduced due to this can be made $O\left(1 / n^{a}\right)$ for any large constant $a>0$, which does not change our analysis.

${ }^{8}$ Say, $S=10011$, then the number in the interval $[0,1]$ would be 0.10011 (in binary) which converts to 0.59375 in decimal.
}

one decided node (which is needed to satisfy implicit agreement). If there is no decided node, then the candidate nodes have to repeat the process until there is at least one decided node. Therefore, the intuition is to add a verification phase through which the undecided nodes make sure that there is some decided node (if any) and they stop; otherwise all the candidate nodes repeat the process by generating another random number using the shared random bits. Later we see that the bulk of the communication (among the decided and undecided nodes) is in the verification phase. The main idea to keep the messages low and yet achieve the high success probability is by allowing the decided nodes to communicate less (i.e., $o(\sqrt{n})$ ) messages and the undecided nodes to communicate more (i.e., $\omega(\sqrt{n}))$ messages. Since most of the nodes will be decided nodes this reduces the overall communication. Let us now describe the improved algorithm step by step (see Algorithm 1 for pseudocode).

Let us use parameters in the algorithm and later in the analysis, we declare the appropriate value of the parameters by optimizing the message complexity bound.

In the first round, each node elects itself with probability $2 \log n / n$ to become a candidate node. Hence, the size of the candidate node set is $\Theta(\log n)$ with high probability. (Note that modulo choosing this candidate set, the algorithm is Las Vegas.) Similar to the simpler algorithm, the elected candidate nodes are responsible for the agreement. In the next round, each candidate node $v$ samples another $f$ random nodes from the network and gets their values- 0 or 1 . Then each candidate node $v$ computes $p(v)$ as the fraction of 1 s it receives among the $f$ samples. It will be shown that, with high probability, all the $p(v)$ values belong to the strip of length $\delta=\sqrt{24 \log n / f}$. Now we iterate the following steps until the agreement succeeds. The candidate nodes generate a common random real number, say $r$, in the interval $[0,1]$, using the global coin. All the candidate nodes $v$ locally check if the difference $|p(v)-r|$ is more than $4 \delta$. For the nodes whose difference is more than $4 \delta$, they become decided nodes; otherwise, they become undecided nodes. Then, all the deciding nodes decide on 0 if their $p(v)$ is on the left side of $r$ in the line segment [0,1]; otherwise, they decide on 1. Then the algorithm performs a verification to make sure that there exists at least one decided node. If there exists at least one decided node, then the algorithm stops; otherwise it repeats the iteration.

In the verification phase, each undecided node tries to check if there is at least one decided node; if so, it can safely terminate. In this phase, all the decided nodes (if there are any) sample $2 n^{1 / 2-\gamma} \sqrt{\log n}$ random nodes (where $\gamma$ is a suitably chosen parameter that will be fixed in the analysis to optimize the message complexity) from the $n$ nodes and send them the message $\langle$ decided,deciding_value $\rangle$. Similarly, in parallel, all the undecided nodes (if there are any) sample $2 n^{1 / 2+\gamma} \sqrt{\log n}$ random nodes and send them the message $\langle$ undecided $\rangle$. Then it can be shown that for any pair of decided and undecided nodes, with high probability, there is at least one common node in their randomly sampled set. The common neighbor informs the undecided node about the existence of the decided node. Therefore, if there is any decided node, the undecided node knows the existence of the decided node(s) and their decided value. Thus the agreement is solved with all the candidate nodes (decided and 
undecided) having the agreed value. Below we show all the above claims formally.

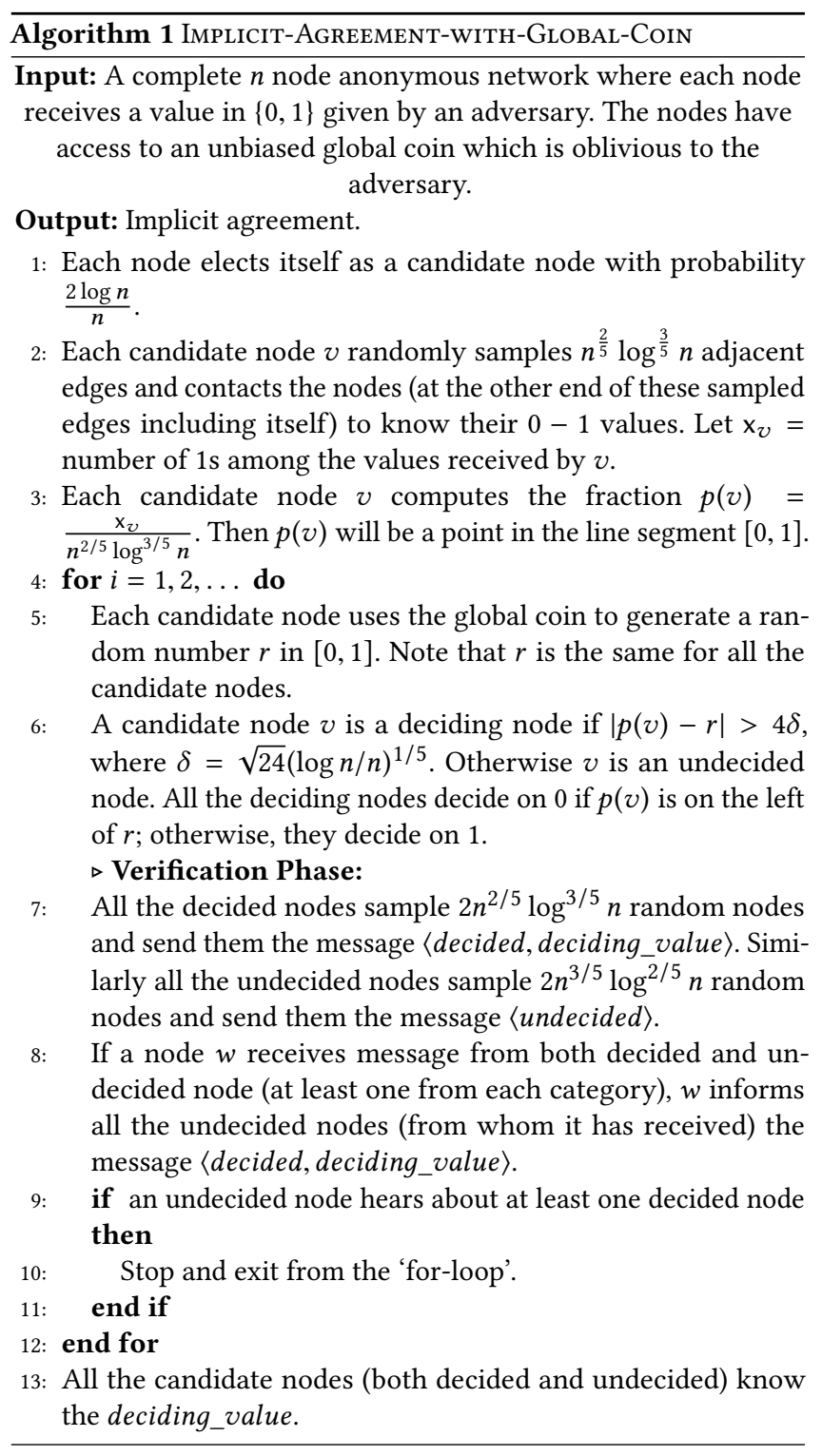

Let us first show that if the number of random samples is $f$, then the strip length is $O(\sqrt{\log n / f})$ whp.

LEMma 3.1. Suppose the values $\{0,1\}$ are distributed arbitrarily over the n-nodes. Let $f$ be the number of random samples drawn by each candidate node. Then the $p(v)$ value of all the candidate nodes $v$ belong to a strip of length $\delta=\sqrt{24 \log n / f}$ with high probability.

Proof. Each candidate node samples $f$ nodes from the set of $n$ nodes uniformly and independently at random. Consider an arbitrary but fixed candidate node $v$. Let $X_{1}, \ldots, X_{f}$ be the $0-1$ random variable corresponding to the $f$ random samples by the node $v$. Notice that each $X_{i}$ is independent and identically distributed $0-1$ random variable. Hence, the expected value $E\left(X_{i}\right)$ is same for all
$X_{i}$, say $E\left(X_{i}\right)=\mu$. By the definition of $p(v), p(v)=\left(\sum_{i=1}^{f} X_{i}\right) / f$. Now we use the following $(\epsilon, \alpha)$-approximation result for $\mu$.

Lemma 3.2 (Theorem 11.1, [20]). Let $X_{1}, X_{2}, \ldots, X_{m}$ be independent and identically distributed indicator random variables, with $\mu=E\left[X_{i}\right]$. If $m \geq(3 \ln (2 / \alpha)) / \epsilon^{2} \mu$, then

$$
\operatorname{Pr}\left(\left|\frac{1}{m} \sum_{i=1}^{m} X_{i}-\mu\right| \geq \epsilon \mu\right) \leq \alpha
$$

Therefore, we get $\operatorname{Pr}(|p(v)-\mu| \geq \epsilon \mu) \leq \alpha$, where the number of samples $f$ should be larger than $(3 \ln (2 / \alpha)) / \epsilon^{2} \mu$. Let us choose $f=(3 \ln (2 / \alpha)) / \epsilon^{2} \mu$ and $\alpha=2 / n^{2}$. Then $p(v) \in(\mu-\epsilon \mu, \mu+\epsilon \mu)$ with probability at least $\left(1-2 / n^{2}\right)$. That is, with high probability, $p(v)$ belongs to the strip of length $2 \epsilon \mu$. Now we have,

$$
\begin{aligned}
f & =\left(3 \ln \left(n^{2}\right)\right) / \epsilon^{2} \mu=(6 \ln (n)) / \epsilon^{2} \mu \\
\Rightarrow \epsilon & =\sqrt{6 \ln (n) / f \mu} \\
\Rightarrow 2 \epsilon \mu & =\sqrt{24 \ln (n) \mu / f} \leq \sqrt{24 \ln (n) / f}, \quad[\text { since } \mu \leq 1]
\end{aligned}
$$

Therefore, the strip length is at most $\sqrt{24 \ln (n) / f}$ with high probability. Since $v$ is an arbitrary node, the same is true for all the candidate nodes. Moreover, $\sqrt{24 \ln (n) / f}<\sqrt{24 \log n / f}$, as $\ln (x)<$ $\log (x)^{9}$. Hence the lemma.

Claim 3.3. Suppose $v$ is a decided node and $u$ is an undecided node. If $v$ samples $2 n^{1 / 2-\gamma} \sqrt{\log n}$ random nodes and $u$ samples $2 n^{1 / 2+\gamma} \sqrt{\log n}$ random nodes for any $\gamma>0$, then, with high probability, there is at least one common node in their sampled set.

Proof. Let $A$ be the set of random nodes sampled by $v$ and similarly the set $B$ sampled by $u$. Then $|A|=2 n^{1 / 2-\gamma} \sqrt{\log n}$ and $|B|=2 n^{1 / 2+\gamma} \sqrt{\log n}$. The probability that any node will be in $A$ is $|A| / n$, since $A$ is chosen uniformly at random over $n$ nodes. Therefore, the probability that a particular node of $B$ will not be in $A$ is $(1-|A| / n)$. Hence the probability that no node of $B$ will be in $A$, i.e., $\operatorname{Pr}[A \cap B=\emptyset]$ is $(1-|A| / n)^{|B|}$. It gives,

$$
\left(1-\frac{2 n^{1 / 2-\gamma} \sqrt{\log n}}{n}\right)^{2 n^{1 / 2+\gamma} \sqrt{\log n}} \leq \exp (-4 \log n)=\frac{1}{n^{4}} .
$$

That is $\operatorname{Pr}[A \cap B \neq \emptyset]>\left(1-1 / n^{4}\right)$. In other words, with high probability, there is a common member in the sampled set of $v$ and $u$.

Lemma 3.4. For any pair of decided and undecided nodes, with high probability there is at least one common node in their random sampled set.

Proof. The proof follows from the above Claim 3.3 and by taking a union bound over all the candidate nodes. Recall that there is at most $O(\log n)$ candidate nodes.

Now let us calculate the message complexity by putting the appropriate value of the parameters.

\footnotetext{
${ }^{9} \log (x)$ is the logarithmic function with base 2 and $\ln (x)$ is the natural logarithmic function, i.e., with base $e$.
} 
LeMmA 3.5. The message complexity of the Algorithm 1 is $O\left(n^{\frac{2}{5}} \log ^{\frac{8}{5}} n\right)$ in expectation and also holds with probability at least $\left(1-1 / n^{\frac{1}{5}}\right)$.

Proof. In the first phase, every candidate node contacts $f$ nodes to compute $p(v)$. So the messages cost in this part of the algorithm is $O(f \log n)$ as size of the candidate nodes is $O(\log n)$. In the second phase (i.e., the verification phase), all the decided nodes sample $2 n^{1 / 2-\gamma} \sqrt{\log n}$ nodes. There could be at most $O(\log n)$ decided nodes (say, all the candidate nodes). The undecided nodes sample $2 n^{1 / 2+\gamma} \sqrt{\log n}$. Similarly there could be at most $O(\log n)$ undecided nodes. The probability of the event that there exist undecided nodes is $4 \delta$ which is $4 \sqrt{24 \log n / f}$. Hence the message cost of the verification part in a single iteration is $O\left(\log n\left(2 n^{1 / 2-\gamma} \sqrt{\log n}+\right.\right.$ $\left.\left.(4 \sqrt{24 \log n / f}) \cdot\left(2 n^{1 / 2+\gamma} \sqrt{\log n}\right)\right)\right)$. We might have to repeat the process from the verification phase if there is no decided node. The probability that there is no decided node in one iteration is at most $4 \delta$. This implies the probability that there is at least one decided node in one iteration is at least $(1-4 \delta)$. Since the iterations are independent, the number of repetitions to get high probability of success is at most $1 /(1-4 \delta)=O(1)$, since $\delta=1 / n^{1 / 5}$. Thus, the verification phase is repeated at most $O(1)$ times. Hence, to achieve agreement with high probability of success, the total number of messages used by the Algorithm 1 is:

$$
\begin{aligned}
& O\left(f \log n+2 n^{\left(\frac{1}{2}-\gamma\right.} \log ^{3 / 2} n+8 \log ^{2} n(\sqrt{24 / f}) n^{\frac{1}{2}+\gamma}\right) \\
& =O\left(f \log n+n^{\frac{1}{2}-\gamma} \log ^{3 / 2} n+\frac{n^{\frac{1}{2}+\gamma}}{\sqrt{f}} \log ^{2} n\right)
\end{aligned}
$$

By optimizing the above quantity for the parameters $\gamma$ and $f$, we get:

$$
\gamma=\frac{1}{10}-\frac{1}{5} \log _{n}(\sqrt{\log n}) \text { and } f=n^{\frac{2}{5}} \log ^{\frac{3}{5}} n .
$$

Furthermore, the above value of $\gamma$ and $f$ gives the precise value of the following quantities.

- The strip length: $\delta=\sqrt{24 \log n / f}=\sqrt{24}(\log n / n)^{1 / 5}$, (cf. Lemma 3.1).

- In the verification phase, number of sampled nodes by the decided nodes: $2 n^{1 / 2-\gamma} \sqrt{\log n}=2 n^{2 / 5} \log ^{3 / 5} n$.

- In the verification phase, number of sampled nodes by the undecided nodes: $2 n^{1 / 2+\gamma} \sqrt{\log n}=2 n^{3 / 5} \log ^{2 / 5} n$.

Since each undecided node sends $O\left(n^{3 / 5} \log ^{2 / 5} n\right)$ messages (which is $\left.\omega\left(n^{\frac{2}{5}} \log ^{\frac{8}{5}} n\right)\right)$ and the probability that there exist undecided nodes is $4 \sqrt{24 \log n / f} \leq n^{-\frac{1}{5}}$, the message complexity of the Algorithm 1 is $O\left(n^{\frac{2}{5}} \log ^{\frac{8}{5}} n\right)$ with probability at least $\left(1-n^{-\frac{1}{5}}\right)$. The same message bound also holds in expectation, since the verification phase is repeated $O(1)$ times.

Correctness of the Agreement. Finally we claim that the Algorithm 1 outputs valid and correct implicit agreement. All the decided nodes agree on the same value. The size of the candidate node set is $\Theta(\log n)$ with high probability. If all the values in the initial distribution are 0 (or 1 respectively) then $p(v)$ of all the candidate nodes will be 0 (or 1 respectively) and they all agree on the same $p(v)$ value with high probability. Moreover, if the generated random number $r$ (using the global coin) doesn't fall in the $p(v)$-strip of length $4 \delta$, then all the candidate nodes make a decision and the value of the decision is same. The reason is: if $r$ is outside of the strip then the $p(v)$ for all candidate nodes $v$ are in one side of $r$ in the $[0,1]$ line, i.e., either all are in the left or all are in the right side of $r$. The agreed value is valid as it must be from the input values.

Let us now analyze the time complexity of the algorithm.

Lemma 3.6. The time complexity of the Algorithm 1 is O(1) with high probability.

Proof. Every node tosses a coin with probability $2 \log n / n$ to become a candidate node. Hence, with high probability $O(\log n)$ candidate nodes are elected (easy to show using standard Chernoff bound.This is done locally. Each candidate node contacts $O(\log n)$ random nodes in parallel to compute the $p(v)$ value. This is one time communication and hence takes 1 round. Then algorithm repeats the Step 5, until there is at least one deciding node. This happens when the distance of all the candidate nodes from the random point $r$ is less than $4 \delta$. The probability of this event is at most $4 \delta$. Therefore, the failure probability of the algorithm (i.e., there is no deciding node) is at most $4 \delta$ which is $4 \sqrt{24}(\log n / n)^{1 / 5}$, as $\delta=\sqrt{24}(\log n / n)^{1 / 5}$. Hence there must be one success in $O(1)$ repetitions with high probability. Moreover, inside each repetition, the algorithm takes $O(1)$ rounds as the deciding nodes and undecided nodes sample $f$ nodes in parallel by one time communication. All other computations are done locally. Hence the time complexity is $O(1)$ rounds with high probability.

Therefore, by combining the above Lemma 3.5 and Lemma 3.6, we get the following main result of this section.

THEOREM 3.7. Consider a complete n-node network and CONGEST communication model. There is a randomized algorithm which, with the help of global coin, solves implicit agreement with high probability in $O(1)$ rounds and uses $O\left(n^{\frac{2}{5}} \log ^{\frac{8}{5}} n\right)$ messages in expectation.

\section{SUBSET AGREEMENT}

Suppose a subset $S$ of $k$ nodes (among the $n$ nodes in a complete network) want to accomplish agreement. The nodes in $S$ do not know each other. Further, assume that the size of $S$ is unknown to the nodes. Then one can solve the subset agreement easily using the implicit agreement algorithms in Section 2 and 3. All the $k$ nodes in $S$ act as candidate nodes and run the rest of the implicit agreement algorithm. Since the message complexity of the implicit agreement algorithm is dominated by $O(\mathbf{C} \cdot f)$, where $\mathbf{C}$ is the number of candidate nodes and $f$ is the number of random samples drawn by the candidate nodes, the message complexity of the subset agreement algorithms are $\tilde{O}(k \sqrt{n})$ in the case of accessing private coins only, and $\tilde{O}\left(k n^{0.4}\right)$ in the case of accessing global coin. Moreover, the algorithms finish in $O(1)$ communication rounds.

However notice that, when $k \geq \Omega(\sqrt{n})$ (for the private coins) or when $k \geq \Omega\left(n^{0.6}\right)$ (for global coin), the above message bounds are more than $\Omega(n)$ which is worse than a simple $O(n)$ message complexity algorithm that solves full (explicit) agreement (i.e., $k=$ $n$ ) over all the $n$-nodes. Full agreement can be solved using $O(n)$ messages in $O(1)$ rounds by simply solving implicit agreement (or 
leader election) and the deciding nodes (or the leader) broadcasting the agreed value to all nodes. This is better if $k \geq \sqrt{n}$.

We combine both the above approaches as follows, assuming we know $k$ (which we discuss later). First compute a leader in $S$ using $O\left(\left(k \log ^{3 / 2} n\right) / \sqrt{n}\right)$ messages and $O(1)$ rounds, see [17] (or, see below the leader election algorithm and the analysis). Then the leader notifies its decided_value to all the $n$ nodes in the next round, which takes $O(n)$ messages. Therefore, if the size of the set $S$, i.e., $k$ is known to the nodes of $S$ then the subset agreement problem can be solved with message complexity $O(\min \{k M, n\})$, where $M$ is the message complexity of the implicit agreement algorithm. In fact, it is sufficient if the nodes in $S$ know whether $k$ is less than or greater than $\sqrt{n}$ (less or greater than $n^{0.6}$ for the global coin case).

Let us now discuss an approach to check if the value of $k$ is smaller or larger than $\sqrt{n}$ using $O\left(k \log ^{3 / 2} n\right)$ messages. The similar technique can be used to check whether $k \leq$ or $>n^{0.6}$. Each node in $S$ elects itself with probability $(\log n) / \sqrt{n}$. Then using linearity of expectation and a Chernoff bound, it can be shown that $\Theta((k \log n) / \sqrt{n})$ nodes are elected with high probability. Each elected node again samples another $O(\sqrt{n \log n})$ random referee nodes and sends them its ID. Then it can be shown in the similar way to the proof of Claim 3.3 that any two elected nodes have one common referee with high probability. This implies that any elected node has at least one common referee among all the other elected nodes. In the next round, each referee node sends back the count of the IDs to the elected nodes from where it received the IDs. Then it is easy to see that: if the elected nodes get back $\Omega(\log n)$ count, then $k \geq \Omega(\sqrt{n})$; else if the count is $o(\log n)$, then $k \leq o(\sqrt{n})$. Thus the elected nodes can distinguish whether the size of $S$ is $o(\sqrt{n})$ or $\Omega(\sqrt{n})$. With this knowledg (i.e., whether $k$ is $\Omega\left(n^{0.5}\right)$ or not) the nodes in $S$ can run either the $O(k M)$ message complexity algorithm (if $k \leq o(\sqrt{n})$ ), or the linear message complexity algorithm (if $k \geq \Omega(\sqrt{n})$ ). The message complexity of this size estimation approach is $\Theta((k \log n) / \sqrt{n}) \cdot O(\sqrt{n \log n})$, which is $O\left(k \log ^{3 / 2} n\right)$.

There is still a small issue with the above idea: only the elected nodes of $S$ may know about the value of $k$ and not all the nodes in $S$ are elected. So how do the non-elected nodes also know the condition that $k \leq o(\sqrt{n})$, so that all the nodes in $S$ can run the implicit agreement algorithm (as a candidate node)? Note that when $k \geq \Omega(\sqrt{n})$, the non-elected nodes do not need to know the condition as the algorithm sends the decided_value to all the $n$ nodes (explicit agreement) in the next round. When $k \leq o(\sqrt{n})$, all the nodes in $S$ wait for some constant number rounds i.e., until the run time of algorithm when $k \geq \Omega(\sqrt{n})$. If the non-elected nodes receive any message (decided_value) by that time, then it's understood that $k \geq \Omega(\sqrt{n})$ (that's when all the $n$ nodes received the agreement value). Otherwise, if the non-elected nodes do not hear anything by that time, it indicates that $k \leq o(\sqrt{n})$ and all the nodes in $S$ will start executing the implicit agreement algorithm in the next round onwards.

Then we get the two corollaries of the two theorems- (Theorem 2.5) for agreement without global coin i.e., with private coins only and (Theorem 3.7) for agreement with global coin.

Theorem 4.1 (Subset Agreement with Private Coin Only). Consider a complete n-node network $G(V, E)$ and a subset $S \subseteq V$ of size $k$. There is a randomized algorithm which solves the subset agreement over $S$ with high probability in $O(1)$ rounds and uses $\min \{\tilde{O}(k \sqrt{n}), O(n)\}$ messages in expectation.

Theorem 4.2 (Subset Agreement with Global Coin). Consider a complete n-node network $G(V, E)$ and a subset $S \subseteq V$ of size $k$. There is a randomized algorithm which, with the help of global coin, solves the subset agreement over $S$ with high probability and finishes in $O(1)$ rounds and uses $\min \left\{\tilde{O}\left(k n^{0.4}\right), O(n)\right\}$ messages in expectation.

\section{LEADER ELECTION USING A GLOBAL COIN}

Let us first define the (implicit) leader election problem in a general network, while in this section, we consider the problem is a complete network. Given a $n$-node network, the (implicit) leader election is defined as follows.

Definition 5.1 (Implicit Leader Election, [17]). Let every node in the network maintains its status, that can take value in $\{\perp$, Non-ELECTED, ELECTED $\}$. Initially each node starts with status $=$ $\perp$. An implicit leader election satisfies when the final status of exactly one node is ELECTED and all other nodes have status Non-ELECTED. In other words, the elected leader (exactly one) knows that it's the leader and all other nodes know they are not leader.

We show an $\Omega(\sqrt{n})$ lower bound on the message complexity of the leader election problem using shared random bits. There is a $\tilde{O}(\sqrt{n})$ message complexity algorithm which computes a leader in a complete network without shared random bits [17]. The lower bound result demonstrates that having global coin doesn't help to achieve a better message complexity algorithm for the leader election problem compared to what can be acheived using private coins. This results shows that agreement is a strictly easier problem than leader election under access to global coins. More preciselyly, we show that any algorithm $\mathcal{A}$ which computes a leader with probability $\epsilon$, for a fixed constant $0<\epsilon<1$, must incur $\Omega(\sqrt{n})$ messages in a complete network. The message size could be arbitrarily large. That is, the result holds in both LOCAL and CONGEST models. Kutten et al. [17] showed a $\Omega(\sqrt{n})$ lower bound on the leader election problem without the global coin. We adapt the proof framework from there and show the same lower bound holds on the number of messages when using shared random bits. The model assumes that all the processors have an access to an unbiased global coin through which they can generate common random bits. Further, all processors run the same algorithm. We show the lower bound result by first assuming the nodes are anonymous. Then it can be easily extended (using standard method) to the non-anonymous case where each node has unique identifiers. The main result is presented in Theorem 5.2. We defer the detailed proof to the full version.

Theorem 5.2. Consider any algorithm $\mathcal{A}$ that has an access to an unbiased random coin and sends at most $f(n)$ messages (of arbitrary size) with high probability in a complete network of $n$ nodes. If $\mathcal{A}$ solves leader election with probability at least $\epsilon$, for some large enough constant $0<\epsilon<1$, then $f(n) \in \Omega(\sqrt{n})$. This holds even if nodes are equipped with unique identifiers (chosen by an adversary).

REMARK 5.3. We like to mention that the above theorem is tight with respect to the number of messages and success probability of 
electing a leader. Notice that a naive randomized algorithm (where each node chooses to become leader with probability $1 / n$ and then terminates) computes a leader with zero message cost, but the success probability is $\left(\begin{array}{l}n \\ 1\end{array}\right)(1 / n)(1-1 / n)^{n-1} \approx 1 /$ e. Moreover, there is an algorithm which succeeds with large constant probability but incurs $\Omega(\sqrt{n})$ messages [17]. This implies that there is a sudden jump in the message complexity when breaking the 1/e barrier in success probability.

\section{CONCLUSION}

We studied the role played by randomization in the message complexity of randomized agreement. Some open questions raised by our work: (1) Is our upper bound of $\tilde{O}\left(n^{0.4}\right)$ tight (up to polylog factors) for agreement with a global coin? Can we show a lower bound on agreement with global coins? (2) Can we show an agreement algorithm that works with a common coin which is weaker than a (unbiased) global coin assumed here? (3) Can we improve our upper bounds for subset agreement and show lower bounds? (4) Can we extend our results for general graphs? (5) Finally, what are the message bounds for agreement and leader election in the presence of Byzantine nodes?

\section{REFERENCES}

[1] Y. Afek and E. Gafni. 1991. Time and Message Bounds for Election in Synchronous and Asynchronous Complete Networks. SIAM f. Comput. 20, 2 (1991), 376-394. https://doi.org/10.1137/0220023 arXiv:http://epubs.siam.org/doi/pdf/10.1137/0220023

[2] A. Agbaria and R. Friedman. 2003. Overcoming Byzantine Failures Using Checkpointing. University of Illinois at Urbana-Champaign Coordinated Science Laboratory technical report no. UILU-ENG-03-2228 (CRHC-03-14) (2003).

[3] Yair Amir, Claudiu Danilov, Jonathan Kirsch, John Lane, Danny Dolev, Cristina Nita-Rotaru, Josh Olsen, and David John Zage. 2006. Scaling Byzantine FaultTolerant Replication toWide Area Networks. In Proc. of International Conference on Dependable Systems and Networks (DSN). 105-114.

[4] D. P. Anderson and J. Kubiatowicz. 2002. The worldwide computer. Scientific American 286, 3 (2002), 28-35.

[5] Hagit Attiya and Jennifer Welch. 2004. Distributed Computing: Fundamentals, Simulations and Advanced Topics (2nd edition). John Wiley Interscience.

[6] Michael Ben-Or. 1983. Another Advantage of Free Choice: Completely Asynchronous Agreement Protocols. In Proc. of the Second Annual ACM SIGACT-SIGOPS Symposium on Principles of Distributed Computing (PODC). 27-30.

[7] Michael Ben-Or and Nathan Linial. 1989. Collective Coin Flipping. Advances in Computing Research 5 (1989), 91-115.

[8] Michael Ben-Or, Elan Pavlov, and Vinod Vaikuntanathan. 2006. Byzantine agreement in the full-information model in $\mathrm{O}(\log \mathrm{n})$ rounds. In Proc. of the 38th Annual ACM Symposium on Theory of Computing (STOC). 179-186.
[9] Miguel Castro and Barbara Liskov. 2002. Practical byzantine fault tolerance and proactive recovery. ACM Trans. Comput. Syst. 20, 4 (2002), 398-461.

[10] Pesech Feldman and Silvio Micali. 1997. An Optimal Probabilistic Protocol for Synchronous Byzantine Agreement. SIAM 7. Comput. 26, 4 (1997), 873-933.

[11] P Humblet. 1984. Electing a leader in a clique in $O(n \log n)$ messages. (1984). Intern. Memo., Laboratory for Information and Decision Systems, M.I.T., Cambridge, Mass.

[12] Valerie King and Jared Saia. 2011. Breaking the $O\left(n^{2}\right)$ bit barrier: Scalable byzantine agreement with an adaptive adversary. F. ACM 58, Article 18 (July 2011), 24 pages. Issue 4.

[13] E. Korach, S. Kutten, and S. Moran. 1990. A modular technique for the design of efficient distributed leader finding algorithms. ACM Trans. Program. Lang. Syst. 12, 1 (Jan. 1990), 84-101. https://doi.org/10.1145/77606.77610

[14] E. Korach, S. Moran, and S. Zaks. 1987. The Optimality of Distributive Constructions of Minimum Weight and Degree Restricted Spanning Trees in a Complete Network of Processors. SIAM f. Comput. 16, 2 (1987), 231-236. https: //doi.org/10.1137/0216019 arXiv:http://epubs.siam.org/doi/pdf/10.1137/0216019

[15] E. Korach, S. Moran, and S. Zaks. 1989. Optimal lower bounds for some distributed algorithms for a complete network of processors. Theor. Comput. Sci. 64, 1 (1989), 125 - 132. https://doi.org/10.1016/0304-3975(89)90103-5

[16] Shay Kutten, Gopal Pandurangan, David Peleg, Peter Robinson, and Amitabh Trehan. 2015. On the Complexity of Universal Leader Election. F. ACM 62, 1 (2015), 7:1-7:27.

[17] Shay Kutten, Gopal Pandurangan, David Peleg, Peter Robinson, and Amitabh Trehan. 2015. Sublinear bounds for randomized leader election. Theor. Comput. Sci. 561 (2015), 134-143.

[18] Nancy Lynch. 1996. Distributed Algorithms. Morgan Kaufman Publishers, Inc., San Francisco, USA.

[19] Dahlia Malkhi and Michael K. Reiter. 1997. Unreliable Intrusion Detection in Distributed Computations. In Proc.of the 10th Computer Security Foundations Workshop (CSFW). 116-125.

[20] Michael Mitzenmacher and Eli Upfal. 2017. Probability and Computing: Randomization and Probabilistic Techniques in Algorithms and Data Analysis (second ed.). Cambridge University Press.

[21] Rajeev Motwani and Prabhakar Raghavan. 1995. Randomized Algorithms. Cambridge University Press.

[22] Gopal Pandurangan. 2018. Distributed Network Algorithms. http://sites.google. com/site/gopalpandurangan/dnabook.pdf

[23] Marshall C. Pease, Robert E. Shostak, and Leslie Lamport. 1980. Reaching Agreement in the Presence of Faults. F. ACM 27, 2 (1980), 228-234.

[24] David Peleg. 2000. Distributed Computing: A Locality-Sensitive Approach. SIAM, Philadelphia.

[25] Michael O. Rabin. 1983. Randomized Byzantine Generals. In Proc. of the 24th Annual Symposium on Foundations of Computer Science (FOCS). 403-409.

[26] Sean C. Rhea, Patrick R. Eaton, Dennis Geels, Hakim Weatherspoon, Ben Y. Zhao, and John Kubiatowicz. 2003. Pond: The OceanStore Prototype. In Proc. of the FAST '03 Conference on File and Storage Technologies. 1-14.

[27] Elaine Shi and Adrian Perrig. 2004. Designing secure sensor networks. IEEE Wireless Commun. 11, 6 (2004), 38-43.

[28] Alex Wright. 2009. Contemporary approaches to fault tolerance. Commun. ACM 52, 7 (2009), 13-15.

[29] Hiroyuki Yoshino, Naohiro Hayashibara, Tomoya Enokido, and Makoto Takizawa. 2005. Byzantine Agreement Protocol using Hierarchical Groups. In Proc.of the 11th International Conference on Parallel and Distributed Systems (ICPADS). 64-70. 